\title{
Evaluación del valor protector de los trabajos de defensa del núcleo Canfranc-Estación
}

\author{
Protective value evaluation of defense work \\ in Canfranc-Estación settling
}

\author{
Esteban-Muñoz, A. ${ }^{* *}$; García-Robredo, F. ${ }^{2}$; Robredo, J.C. ${ }^{2}$ \\ ${ }^{1}$ Departamento de Geología, Facultad de Ciencias Ambientales y Bioquímicas, Campus de Toledo, \\ Universidad de Castilla la Mancha, España. \\ ${ }^{2}$ Departamento de Ingeniería y Gestión Forestal y Ambiental, \\ Escuela Técnica Superior de Ingeniería de Montes, Forestal y del Medio Natural, \\ Campus de Ciudad Universitaria, Universidad Politécnica de Madrid, España
}




\section{Resumen}

El cálculo del valor protector de obras de defensa como externalidad positiva resulta clave en aspectos como la ratificación económica o la toma de decisiones de conservación de las obras. Las obras de protección hidrológico-forestales situadas en Canfranc-Estación suponen un claro ejemplo de éxito que supera los 100 años de historia, pero que carece de validación económica y que se encuentra en paulatino deterioro. Con el objetivo de dar argumentos para mantener estas obras de protección se ha calculado su valor protector actual. Además, se han obtenido datos relevantes relativos al riesgo presente en Canfranc-Estación, afectado históricamente por inundaciones, por aludes y por caída de bloques.

Se han integrado y adaptado diferentes herramientas de valoración económica con base en el Método de los Costes Evitados. Para aplicar esta metodología se ha calculado el riesgo cuantitativo (RC) de los dos peligros principales-aludes e inundaciones -, descartando un tercer peligro - desprendimiento de bloques - por insuficiente relevancia. Ambos peligros se han modelado en dos escenarios: actual (S1) y sin obras (S2), en los cuales se han calculado los costes del proyecto (en S1) y el daño generado por cada peligro (en S1 y en S2). Los indicadores económicos e indicadores del riesgo verifican la alta rentabilidad y el alto valor protector de estas obras. Además, se han estimado los indicadores económicos de un hipotético proyecto consistente en realizar la misma obra en la actualidad, revelando la ventaja que pueden presentan este tipo de obras a largo plazo.

Palabras clave: Valor Actual Neto, IBER, RAMMS::AVALANCHE, Mantenimiento, Cuencas vertientes de montaña, Los Arañones.

\section{Summary}

Protective value evaluation in defense works is a required positive externality for economic validation or for decision making. Canfranc-Estación's hydrological-forestry works are a clearly 100 years old case of success. However, these works do not have economic validation and they are suffering a gradual spoilage. Therefore, the objective is to provide arguments to maintain these works. Protective value results and indirect risk results, obtained in protective value calculation process, make it possible.

Different economic valuation tools have been integrated in the main tool called Damage Cost Avoided. To apply this methodology, the quantitative risk for two mainly dangers - avalanches and streams

flows - have been calculated. A third danger - block detachments - has been discarded due to lack of relevance. Two mainly dangers have been modelled in two scenarios: current (S1); and without works (S2). Costs (in S1) and damages (in S1 and S2) have been obtained. Mainly results have been summarized in economic indicators and risks indicators. Both show high profitability and high protective value. In addition, economic indicators of a hypothetical project, which consists of building the same defense works with current costs, have been estimated. Result show that building the protection works make a long-term advantage.

Keywords: Net Current Value, IBER, RAMMS::AVALANCHE, Maintenance, Mountain watersheds, Los Arañones. 


\section{Introducción}

\subsection{Reseña histórica hidrológico-forestal}

El núcleo poblacional de Canfranc-Estación nace a finales del siglo XIX con la decisión de la Comisión Internacional de los Ferrocarriles Transpirenaicos de establecer una estación internacional en el único valle viable del Pirineo central. En una zona pobre en recursos, cuya fuente de ingresos era su situación fronteriza (Madoz, 1846-1850; Benito, 1998), se desarrolló un núcleo en torno a la estación. No obstante, al sufrir alto riesgo por peligros naturales, mientras comenzaba la construcción de la estación en 1908, la Sexta División Hidrológico-Forestal comenzó la mayor obra de corrección y defensa hidrológico-forestal (Fig. l y Fig. 2) efectuada hasta la fecha en el ámbito español (Ayerbe, 1912b; Bernad, 1913; Azpeitia, 1919; Azpeitia, 1922b; Ayerbe, 1952; Benito, 1998; Pemán y Pérez-Soba, 2013; Fábregas Reigosa, 2015). Al ser una obra de una disciplina reciente se aplicaron diversas medidas - alguna genuina - para corregir:

1. Los desprendimientos de bloques, mediante regeneración y repoblación forestal.

2. Las avenidas torrenciales, mediante fajinas, monte protector, diques de consolidación, diques de retención y canalizaciones.

3. Los aludes, mediante banquetas, puentes de nieve, muros de sujeción, estacadas, muros de tierra y diques vacíos (Ayerbe, 1912a; Bernad, 1913; Azpeitia, 1919; Fábregas y Fábregas, 2001; Pemán y Pérez-Soba, 2013; Fábregas Reigosa, 2015). Durante la fase de construcción se alcanzó un pico poblacional de 2000 habitantes, no vuelto a alcanzar hasta las temporadas invernales actuales (Benito, 1998; Fábregas y Fábregas, 2001). Dicha fase terminó mayormente en 1930 cosechando un gran éxito (González, 1928), aunque se extendió con pequeñas actuaciones hasta años recientes. Desde entonces se han producido aludes importantes, pero las obras han quedado relegadas a un segundo plano, actuando únicamente de manera reactiva, sin ejecutar labores de mantenimiento y sin tener en cuenta el principio de prudencia (Fábregas y Fábregas, 2001; Ferrando et al., 2004; Leo y Cuchí, 2017; Statham et al., 2018). Esto puede deberse a que existe una visión cualitativa de seguridad (Fábregas Reigosa, 2015). Sin embargo, no existe un dato cuantitativo que demuestre el efecto positivo de las obras ni que permita dar argumentos para invertir en su conservación y gestión.

\subsection{Valor protector}

Previamente, debe entenderse al riesgo, además de como el cruce entre peligro, vulnerabilidad y exposición, como el grado medible en el que, elementos a los que se les asigna valor, están amenazados de dañarse por eventos naturales (Jonkman et 
al., 2003; Fuchs et al.,2007; Cappabianca et al., 2008; Gracia et al., 2014; Fábregas Reigosa, 2015; Geobrugg y Raymar, 2017; Statham et al., 2018). En regiones de montaña los tipos de eventos dañinos aumentan (Fábregas Reigosa, 2015) y, debido a los daños provocados (MAGRAMA, 2013; Statham et al., 2018), existe legislación europea específica desde el siglo XIX (Fábregas Reigosa, 2015; Geobrugg y Raymar, 2017). El efecto reductor del riesgo de las obras de corrección, raramente computado en términos económicos (Pemán y Pérez Soba, 2013; Fábregas Reigosa, 2015), junto con sus costes, se corresponde con el valor protector (Notaro y Paletto, 2004; Notaro y Paletto, 2008; Plan Bleu, 2016).

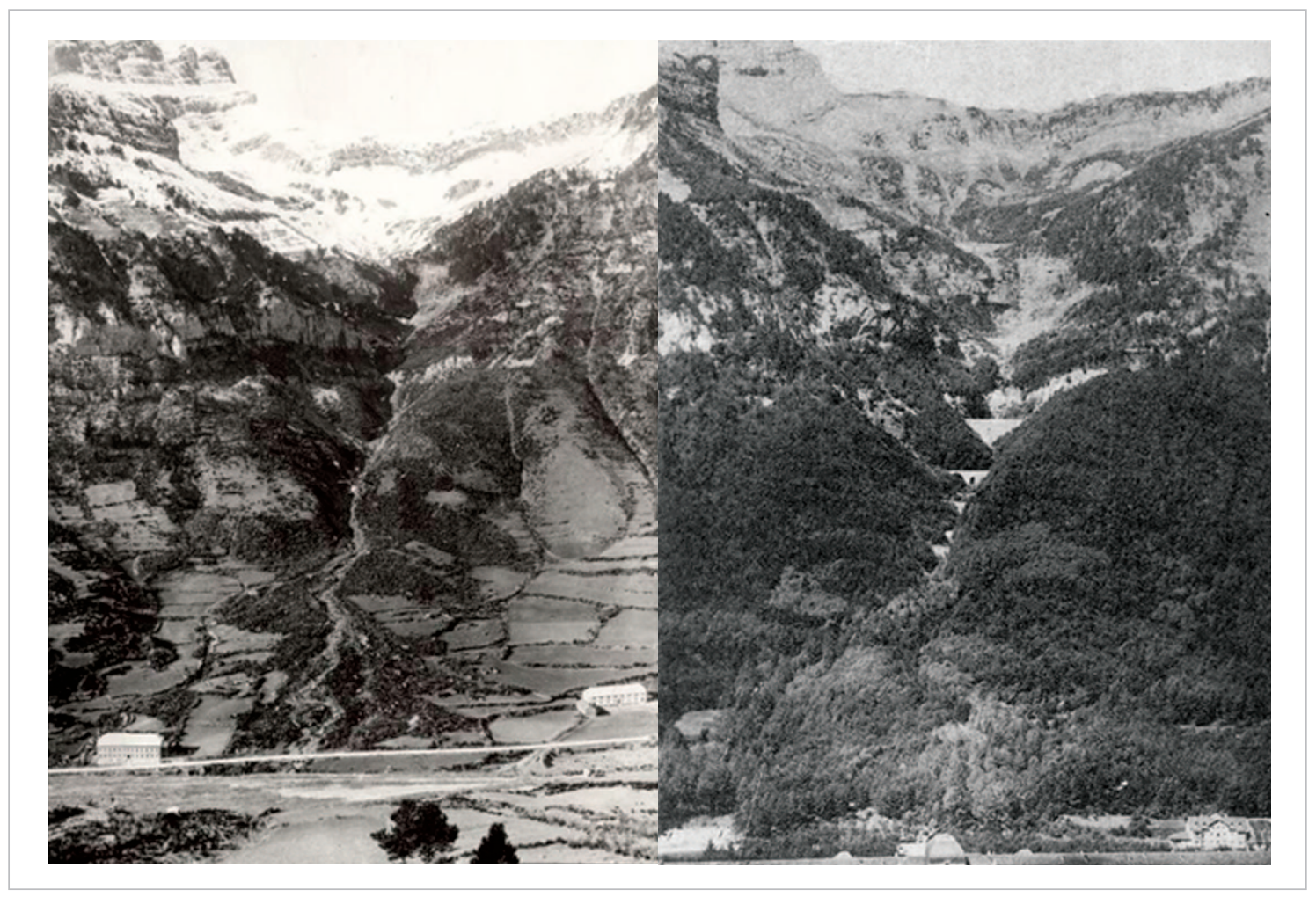

Figura 1. Corrección del torrente Estiviellas antes (izquierda) y después (derecha) de las obras....

Fuente: DARA - Documentos y Archivos de Aragón.

\section{Material y Métodos}

\subsection{Zona de estudio}

Comprende el valle acotado por las cuencas de los torrentes Picauvé, Cargates, San Epifanio, Borreguil de Samán, Rinconada, Estiviellas y Secrás (Canfranc, CCAA de Aragón), de los cuales se han considerado los más relevantes: Estiviellas, Epifanio y Cargates. El valle abarca 814 ha, siendo 769 ha del Monte de Utilidad Pública (MUP) $\mathrm{N}^{\circ} 406$ "Los Arañones" que alberga las obras de defensa (Fig. 2). Presenta un desnivel de 1413 metros (Pico de la Moleta a $2573 \mathrm{msnm}$ ) y la isoterma de los 0 grados entre 


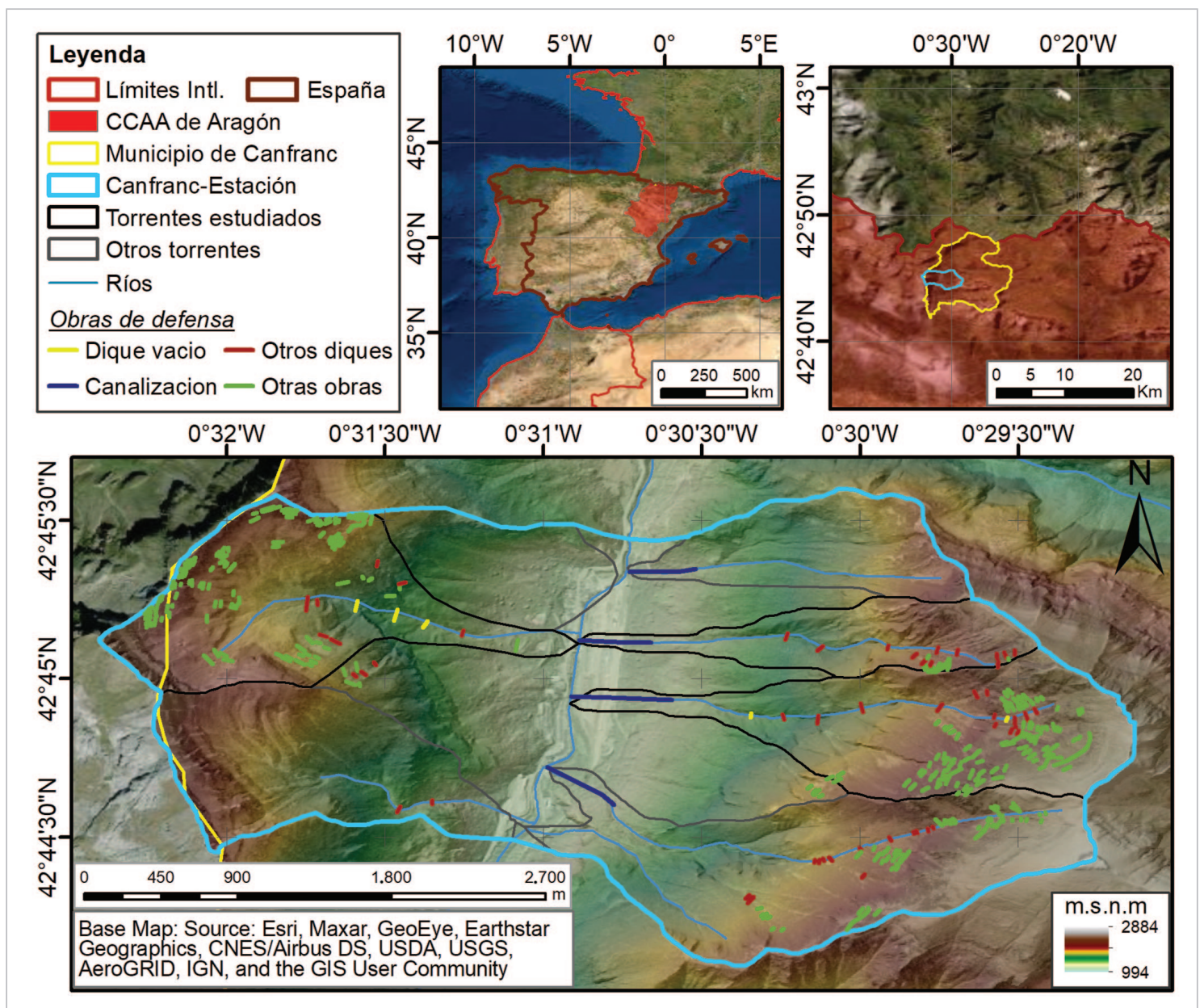

Figura 2. Mapa general. Estiviellas (izquierda), Cargates (derecha superior) y Epifanio (derecha inferior). Fuente: elaboración propia.

los 1500/1600 msnm. El clima es tipo D (Köpen-Geiger) y la vegetación predominante, establecida por la repoblación, se corresponde con la propia de la región bioclimática X(IX)1, destacando el Pinus Sylvestris y el Pinus nigra.

\subsection{Elección y adaptación del método económico}

Las obras han generado efectos positivos, aportando beneficios indirectos (ver 2.2.1), y negativos, suponiendo un coste de establecimiento (CE) y un coste de mantenimiento (CM) (ver 2.2.2). Ambos efectos se han medido para calcular el valor protector. Para los beneficios se ha escogido, entre los métodos posibles (recopilatorio general: Plan Bleu, 2016), el método de los costes evitados complementado con costes de reposición y con el Método del Capital Humano (MCH). Respecto a los costes, se dispone de los CE del proyecto original, mientras que sus $\mathrm{CM}$ se han establecido mediante revisión de precios de mercado (Notaro y Paletto, 2004; Fuchs et al., 2007; Cappabianca et al., 2008; Notaro y Paletto, 2008; Plan Bleu, 2016). Adicionalmente se han calculado los CE y CM de un proyecto hipotético consistente en realizar la misma obra 
con costes actuales. Esta integración de métodos permite calcular indicadores económicos del proyecto (Tab. 1): Valor Actual Neto (VAN), Ratio Coste-Beneficio (RCB) y Plazo de Recuperación de la Inversión (PRI) (fórmulas 1-3). El VAN se corresponde con el valor protector (Fuchs et al., 2007).

\subsubsection{Método de evaluación de daños}

Se requieren las cuatro variables del RC para aludes y avenidas:

1. (A) costes unitarios.

2. (B) peligro.

3. (C) vulnerabilidad.

4. (E) exposición (Cappabianca et al., 2008; Fábregas Reigosa, 2015). El proceso (Tab. 2) consta de tres etapas:

1.- Establecer Periodos de Retorno (PR), calcular sus probabilidades de ocurrencia (P) y calcular los cuantiles de los datos.

2.- Calcular y cruzar las variables del RC para obtener el Daño Esperado (DE).

3.- Calcular el Daño Total Esperado (DTE) para obtener el VAD actualizándolo.

\subsubsection{Método de evaluación de costes}

Los costes se descomponen en CE y CM. Ambos se han calculado mediante la actualización de los costes anuales para obtener el VAC (Tab. 3). Para los CE del proyecto original se han empleado valores del IPC (Instituto Nacional de Estadística) y estimaciones para años anteriores a 1962 (De Motes, 2013), empleando una tasa de conversión de pesetas (Ptas.) a euros (€) de 166.386.

\subsection{Proceso de aplicación del método económico}

El método económico, además de determinar las fórmulas, implica calcular:

1. La reducción del RC en ambos escenarios, que supone tener sus cuatro variables para los peligros de aludes y de avenidas.

2. Los costes CE y CM (ver 2.3.3). La variable peligro se ha obtenido modelan- 
Tabla 1. Cuadro resumen de indicadores económicos.

\begin{tabular}{|c|c|c|c|}
\hline & Indicador & Fórmula & \\
\hline \multirow[b]{3}{*}{$\sum_{1}^{2}$} & $\begin{array}{l}\text { Valor Actual Neto } \\
\text { (S1) }\end{array}$ & $V A N_{S 1}=\sum_{t=0}^{h} \frac{-c_{t}-d_{t}}{(1+i)^{t}}$ & \\
\hline & $\begin{array}{l}\text { Valor Actual Neto } \\
\text { (S2) }\end{array}$ & $\operatorname{VAN}_{S 2}=\sum_{t=0}^{h} \frac{-d_{t}^{\prime}}{(1+i)^{t}}$ & \\
\hline & $\begin{array}{l}\text { Valor Actual Neto } \\
\text { del proyecto } \\
\text { desglosado para } \\
\text { cada escenario }\end{array}$ & $\begin{array}{c}V A N=V A N_{S 1}-V A N_{S 2} \\
=\sum_{t=0}^{n} \frac{\left(d^{\prime}{ }_{a v t}+d^{\prime}{ }_{a l t}\right)-\left[\left(c_{e t}+c_{m t}\right)+\left(d_{a v t}+d_{a l t}\right)\right]}{(1+i)^{t}}= \\
=\sum_{t=0}^{h} \frac{\left(d^{\prime}{ }_{a v t}+d^{\prime}{ }_{a l t}\right)}{(1+i)^{t}}-\sum_{t=0}^{h} \frac{\left(d_{a v t}+d_{a l t}\right)}{(1+i)^{t}}-\sum_{t=0}^{h} \frac{\left(c_{e t}+c_{m t}\right)}{(1+i)^{t}} \\
=\left(V A D_{S 2}-V A D_{S 1}\right)-V A C_{S 1}=V A B-V A C_{S 1}\end{array}$ & [1] \\
\hline 乒 & $\begin{array}{l}\text { Ratio Coste } \\
\text { Beneficio }\end{array}$ & $R C B=\frac{V A C_{S 1}}{\left(V A D_{S 2}-V A D_{S 1}\right)}$ & [2] \\
\hline$\overline{\underline{a}}$ & $\begin{array}{c}\text { Plazo de } \\
\text { Recuperación } \\
\text { de la Inversión }\end{array}$ & Año $t_{r}$ donde se cumple: $V A D_{S 2}-V A D_{S 1}=V A C_{S 1}$ & [3] \\
\hline
\end{tabular}

Donde:

- $\boldsymbol{h}$ Horizonte de planificación.

- $t$ Año cualquiera. $\boldsymbol{t}$ Es el año concreto donde se recupera la inversión.

- $\boldsymbol{c}_{t}$ Costes totales para un año $\boldsymbol{t}$. $\boldsymbol{c}_{\boldsymbol{t} t}$ y $\boldsymbol{c}_{\boldsymbol{m} t}$ se corresponden con el coste de establecimiento de obras para un año $t$ y con el coste de mantenimiento de obras para un año $t$ respectivamente.

- $\boldsymbol{d} t$ Daños totales en el escenario S1 para un año $\boldsymbol{t}$. $\boldsymbol{d}$ avt y $\boldsymbol{d} a t t$ se corresponden con los daños por avenidas y por aludes respectivamente, todos ellos para un año $t$ y en el escenario S1.

- $\boldsymbol{d}^{\prime}{ }_{t}$ Daños totales en el S2 para un año $\boldsymbol{t}$. $\boldsymbol{d}^{\prime}{ }^{\prime}{ }^{\prime}{ }^{\prime} \mathrm{y} \boldsymbol{d}^{\prime}{ }^{\prime}$ at se corresponden con los daños por avenidas y por aludes respectivamente, todos ellos para un año $\boldsymbol{t}$ y en $\mathrm{S} 2$.

- $\boldsymbol{i}$ Tipo de descuento.

- La diferencia entre $\boldsymbol{V} \boldsymbol{A} \boldsymbol{D}_{\mathrm{s} 2} \mathrm{y} \boldsymbol{V} \boldsymbol{A} \boldsymbol{D}_{\mathrm{s} 1}$ son los daños o costes evitados para la sociedad, los cuales se asumen como el valor actual de los beneficios $(\boldsymbol{V A B})$ obtenido por el proyecto.

Hipótesis:

1. Los beneficios y los costes se imputan al final de cada año.

2. Los beneficios y los gastos son conocidos de manera determinista.

3. La inflación de la economía no se tiene en cuenta por utilizar un tipo real de descuento. El tipo de descuento utilizado es del tipo social. Se han realizado los cálculos para intereses entre $1 \%$ y $4 \%$, empleando $2 \%$ como valor principal de referencia (Notaro y Paletto, 2004; Cappabianca et al., 2008). 
Tabla 2. Cuadro resumen del método de evaluación de daños.

\begin{tabular}{|c|c|c|c|}
\hline Etapa & Descripción & Fórmula & \\
\hline 1 & $\begin{array}{l}\text { Probabilidad } \\
\text { de exceder el daño } \\
\text { para un periodo } \\
\text { de retorno concreto } \\
\text { y función } \\
\text { de probabilidad }\end{array}$ & $\begin{array}{c}P\left(D T \geq D T_{P R}\right)=\frac{1}{T} \\
F\left(D T_{P R}\right)=P\left(D T \leq D T_{P R}\right)=1-\frac{1}{T}\end{array}$ & [4] \\
\hline 2 & $\begin{array}{l}\text { Daño Esperado para } \\
\text { PR concretos }\end{array}$ & $\begin{array}{c}D(E)_{P R}^{S}=P_{P R}^{S} * D T_{P R}^{S}=P_{P R}^{S} *\left(\sum_{e=1}^{n} C_{e} * V_{e} * X_{e}\right) \\
C_{v h}=\frac{r}{i} \cdot \frac{(1+i)^{e_{j}-e_{m}}-1}{(1+i)^{e_{j}-e_{m}}}\end{array}$ & [5] \\
\hline 3 & $\begin{array}{c}\text { Daño Total Esperado } \\
\text { y Valor Actual } \\
\text { de los Daños }\end{array}$ & $\begin{array}{c}D T(E)^{S}=R C^{S}=D(E)_{P R_{a}}^{S}+D(E)_{P R_{b}}^{S}+D(E)_{P R_{c}}^{S} \cdots \\
V A D=\frac{D T(E)^{S}}{i} \cdot \frac{(1+i)^{300}-1}{(1+i)^{300}}\end{array}$ & [7] \\
\hline
\end{tabular}

Donde:

- $s$ - Escenario S1 o escenario S2.

- $\boldsymbol{D}(\boldsymbol{E})_{P R}^{S}$ - Daño esperado anual debido a un periodo de retorno $(\boldsymbol{P R})$ en un escenario $(\boldsymbol{s})$.

O $\boldsymbol{P}_{\boldsymbol{P R}}^{S}$ - Probabilidad de ocurrencia $(\mathrm{P})$ de un periodo de retorno $(\boldsymbol{P R})$ en un escenario $(\boldsymbol{s})$.

O $\boldsymbol{D} \boldsymbol{T}_{P R}^{S}$ - Sumatorio de todos los daños generados (DT) por un evento con un periodo de retorno $(\boldsymbol{P R})$ en un escenario $(\boldsymbol{s})$ en función del daño o coste potencial $(\boldsymbol{C})$, de la vulnerabilidad $(\boldsymbol{V})$ y de la exposición $(\boldsymbol{X})$ de cada elemento considerado $(\boldsymbol{e})$.

$\boldsymbol{C}_{\boldsymbol{e}}$ - Coste potencial máximo de rehabilitación o reposición de un elemento " $\boldsymbol{e}$ " cuando este resulta dañado por una determinada intensidad de un evento natural. Se incluye en este coste el daño a vidas humanas " $\boldsymbol{v} \boldsymbol{h}$ ", estimado por el $\mathrm{MCH}$, donde $\boldsymbol{r}$ es la renta media y $\boldsymbol{e}_{\boldsymbol{j}}-\boldsymbol{e}_{\boldsymbol{m}}$ son la edad de jubilación y la edad media respectivamente.

$\boldsymbol{V}_{\boldsymbol{e}}$ - Parámetro que incluye la vulnerabilidad o susceptibilidad de dañarse de un elemento " $\boldsymbol{e}$ ". Se mide en porcentaje de afección y actúa como coeficiente de reducción del coste potencial $\left(\boldsymbol{C}_{\boldsymbol{e}}\right)$. $\boldsymbol{X}_{\boldsymbol{e}}$ - Grado de exposición o probabilidad de ser alcanzado de un elemento " $\boldsymbol{e}$ ". Se mide en probabilidad de ser alcanzado, aunque generalmente se ha empleado una exposición fija de 1 o 0 . Actúa como coeficiente de reducción del coste potencial $\left(\boldsymbol{C}_{\boldsymbol{e}}\right)$.

$\boldsymbol{n}$ - Número de elementos afectados.

- $\boldsymbol{D T}(\boldsymbol{E})^{s}$ - Daño total esperado anual de todos los peligros para un escenario $(\boldsymbol{s})$ en un año cualquiera $\boldsymbol{t}$ y su actualización permite calcular el VAD. Es resultante de la suma de los daños esperados anuales debidos a cada peligro y periodo de retorno $(\boldsymbol{P R})$ contemplado. Además, es el valor empleado para definir el riesgo cuantitativo $\left(\boldsymbol{R} \boldsymbol{C}^{\mathrm{s}}\right)$ de un escenario $(\boldsymbol{s})$.

○ $\boldsymbol{P} \boldsymbol{R}_{a}, \boldsymbol{P} \boldsymbol{R}_{b}, \boldsymbol{P} \boldsymbol{R}_{c}, \ldots$ periodos de retorno contemplados para los cuales se ha calculado el daño esperado anual $\boldsymbol{D}(\boldsymbol{E})$.

- $\boldsymbol{i}$ - Tipo de descuento. 
Tabla 3. Cuadro resumen del método de evaluación de costes.

\begin{tabular}{|c|c|c|}
\hline Descripción & Fórmula & \\
\hline $\begin{array}{l}\text { Valor Actual de los Costes } \\
\text { totales aplicable al proyecto } \\
\text { original y al hipotético }\end{array}$ & $V A C_{S 1}=V A C_{C_{e}}+V A C_{C_{m}}$ & [9] \\
\hline $\begin{array}{l}\text { Costes de Establecimiento } \\
\text { del proyecto original }\end{array}$ & $\begin{array}{c}C_{e a t}=C_{e t}+C_{e t-1}^{\prime} \\
C_{e t}=C_{e t}+C_{e t-1}^{\prime}\left(C_{e t-1} *\left(1+I P C_{t-1}\right)^{1}\right.\end{array}$ & [10] \\
\hline $\begin{array}{l}\text { Costes de Establecimiento } \\
\text { del proyecto hipotético }\end{array}$ & Revisión de precios de mercado & \\
\hline $\begin{array}{l}\text { Costes de Mantenimiento } \\
\text { totales desglosados } \\
\text { empleados para el proyecto } \\
\text { original y para el proyecto } \\
\text { hipotético }\end{array}$ & $\begin{array}{c}V A C_{C_{m}}=C A M a_{S 1}+C A M o_{S 1} \\
C A M a_{S 1}=\frac{C m_{a}}{i} \cdot \frac{(1+i)^{300}-1}{(1+i)^{300}} \\
C A M o_{S 1}=\sum_{t=0}^{h} \frac{C m_{o t}}{(1+i)^{t}}\end{array}$ & {$[11]$} \\
\hline
\end{tabular}

Donde:

- $\boldsymbol{t}$ - Año concreto

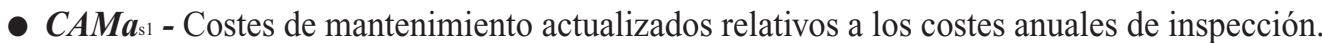

- $\boldsymbol{C M}$ a - Coste anual de las inspecciones.

- $\boldsymbol{C A M o} \boldsymbol{o}_{11}$ - Otros costes de mantenimiento actualizados aplicados cada cierta frecuencia. Para ciertos años $\boldsymbol{t}$ son iguales a cero.

- $\boldsymbol{C} \boldsymbol{m}_{o t}$ - Coste de mantenimiento no anual en un año $\boldsymbol{t}$ concreto.

- $\boldsymbol{C}_{\text {eat }}$ - Coste de establecimiento acumulado de un año $\boldsymbol{t}$ concreto. El $\boldsymbol{V} \boldsymbol{A} \boldsymbol{C}_{\mathrm{Ce}}$ se corresponde con el coste de establecimiento acumulado del último año en el que se realiza la actualización.

- $\boldsymbol{C}_{e t}$ - Coste de establecimiento de un año $\boldsymbol{t}$ concreto.

- $I P C_{t}$ - Valor del IPC para un año $\boldsymbol{t}$ concreto. La actualización de los $\boldsymbol{C}_{e t}$ se realiza acumulando los resultados de año en año.

- $\boldsymbol{i}$ - Tipo de descuento.

Fuente: Elaboración propia.

do aludes con RAMMS::AVALANCHE y avenidas con IBER, mientras que las restantes variables se han obtenido por revisión bibliográfica (ver $2.3 .1 \mathrm{y}$ 2.3.2). En la Fig. 3 se presenta una visión esquemática del proceso.

\subsubsection{Estudio, modelización y valoración de aludes}

Los aludes se clasifican por su formación, velocidad y densidad como inerciales y no inerciales, divididos estos últimos en aludes de placa y de fusión (Mintegui 


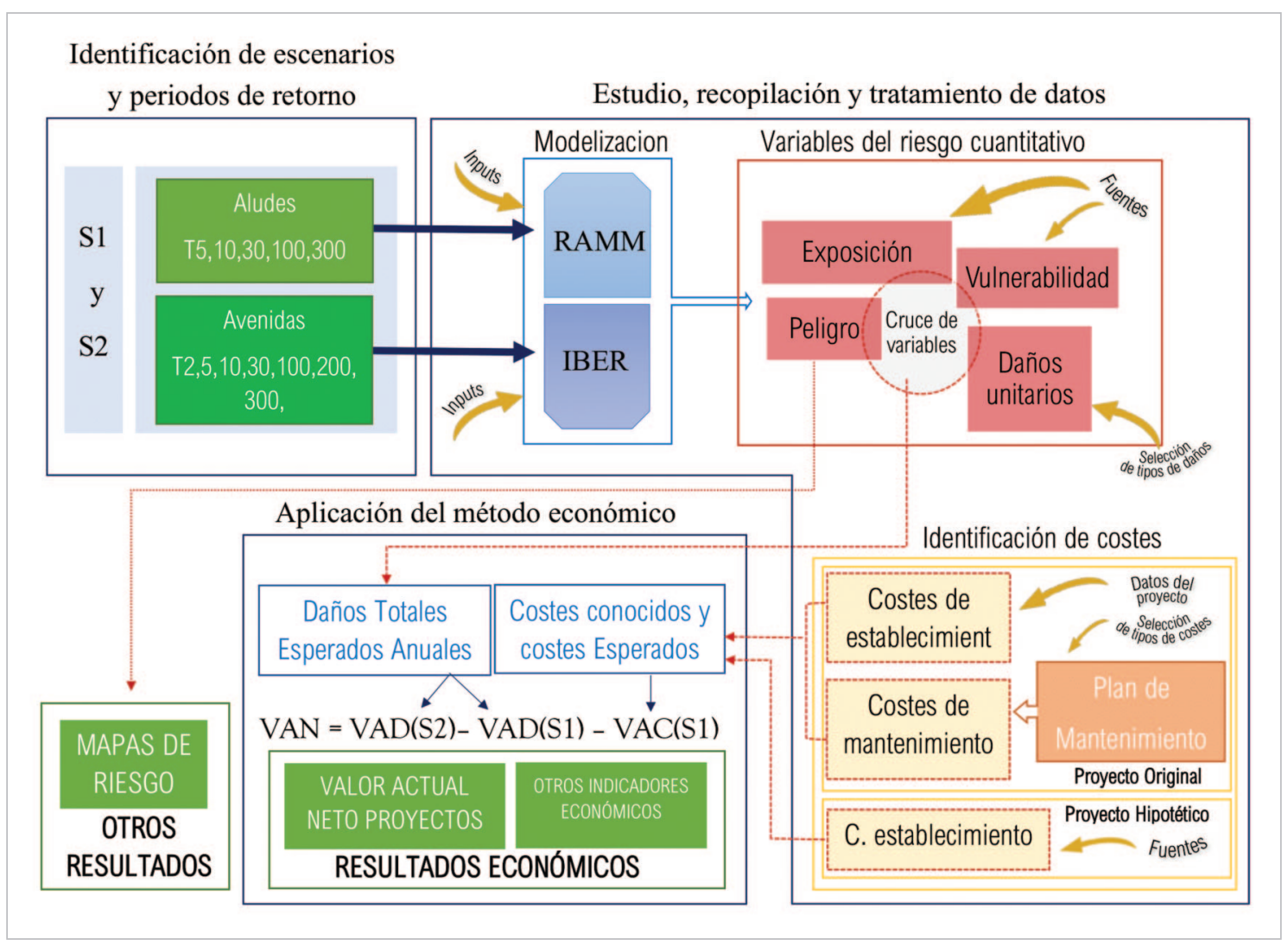

Figura 3. Esquema metodológico general del proceso de valoración de valoración económica.

Fuente: elaboración propia.

et al., 2016; Geobrugg y Raymar, 2017; Leo y Cuchí, 2017). Se ha trabajado asumiendo aludes no inerciales realizando la valoración mediante las variables del RC (Fig. 4).

El peligro se ha caracterizado por la presión, calculada para cada escenario mediante RAMMS:AVALANCHE (Oller et al., 2009; Bartelt et al., 2017). Los escenarios se han adaptado modificando el Modelo Digital del Terreno (MDT), con las características de los diques, (Ayerbe, 1912b; Azpeitia, 1919) y la cubierta forestal con datos históricos (Azpeitia, 1922b; Madoz, 1846-1850). También se han establecido zonas de salida, profundidad y densidad de la nieve (Notaro y Paletto, 2008; Fábregas Reigosa, 2015; Fernández-Santos et al., 2015; Statham et al., 2018; Andrade Fuentes, 2019). La vulnerabilidad de elementos y personas afectadas se ha medido con curvas de vulnerabilidad (Wilhelm, 1998; Cappabianca et al., 2008) y datos bibliográficos (Fuchs et al., 2007; Geobrugg y Raymar, 2017; Statham et al., 2018). La exposición se ha establecido mediante datos bibliográficos (Wilhelm, 1998; Cappabianca et al., 2008; Geobrugg y Raymar, 2017). Los daños unitarios de elementos han sido estimados con costes de reposición y los costes humanos con el MCH (Fuchs et al., 2007; Gracia et al., 2014). Obtenidos todos los datos se han aplicado las fórmulas 4-8. 


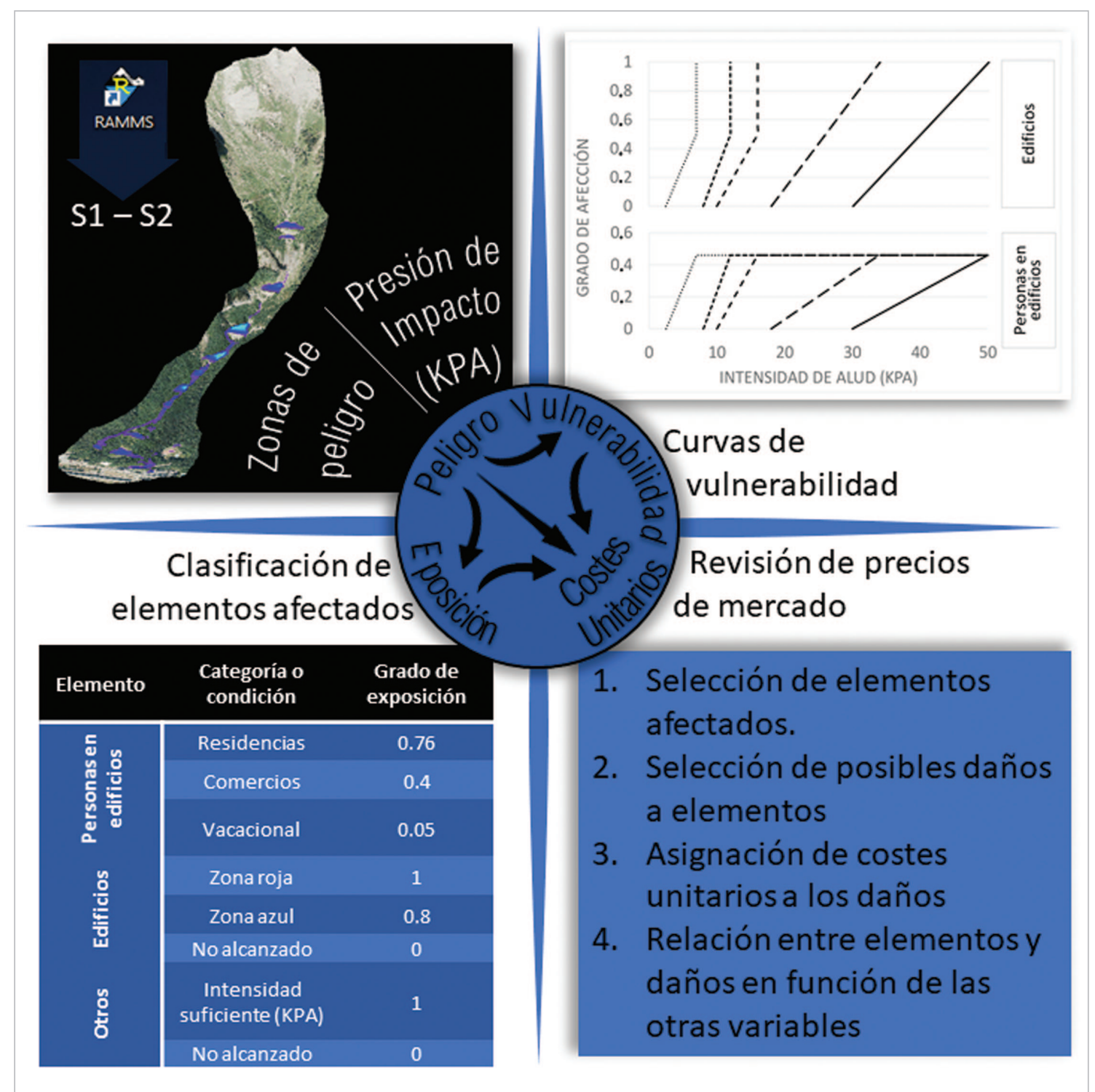

Figura 4. Aplicación de las variables del RC para el peligro de aludes. Curvas de vulnerabilidad reeditadas de Wilhelm (1998) y Cappabianca et al. (2008)

Fuente: elaboración propia.

\subsubsection{Estudio, modelización y valoración de avenidas}

Entre diversas clasificaciones de flujos (Smith, 1986; Pierson et al., 1987; Mintegui et al., 2006; Geobrugg y Raymar, 2017), se puede hablar de flujos bifásicos y monofásicos, divididos estos últimos en flujos hiperconcentrados y flujos de escombros. Sin descartar la existencia de los últimos, se han asumido flujos bifási$\cos$.

Los conceptos relativos a los flujos bifásicos están bien definidos en la legislación y propuestas metodológicas españolas (Gracia et al., 2014; Álvarez et al., 2018; Fernández-Aracil, 2020), resultando de gran aplicabilidad el método de valoración 


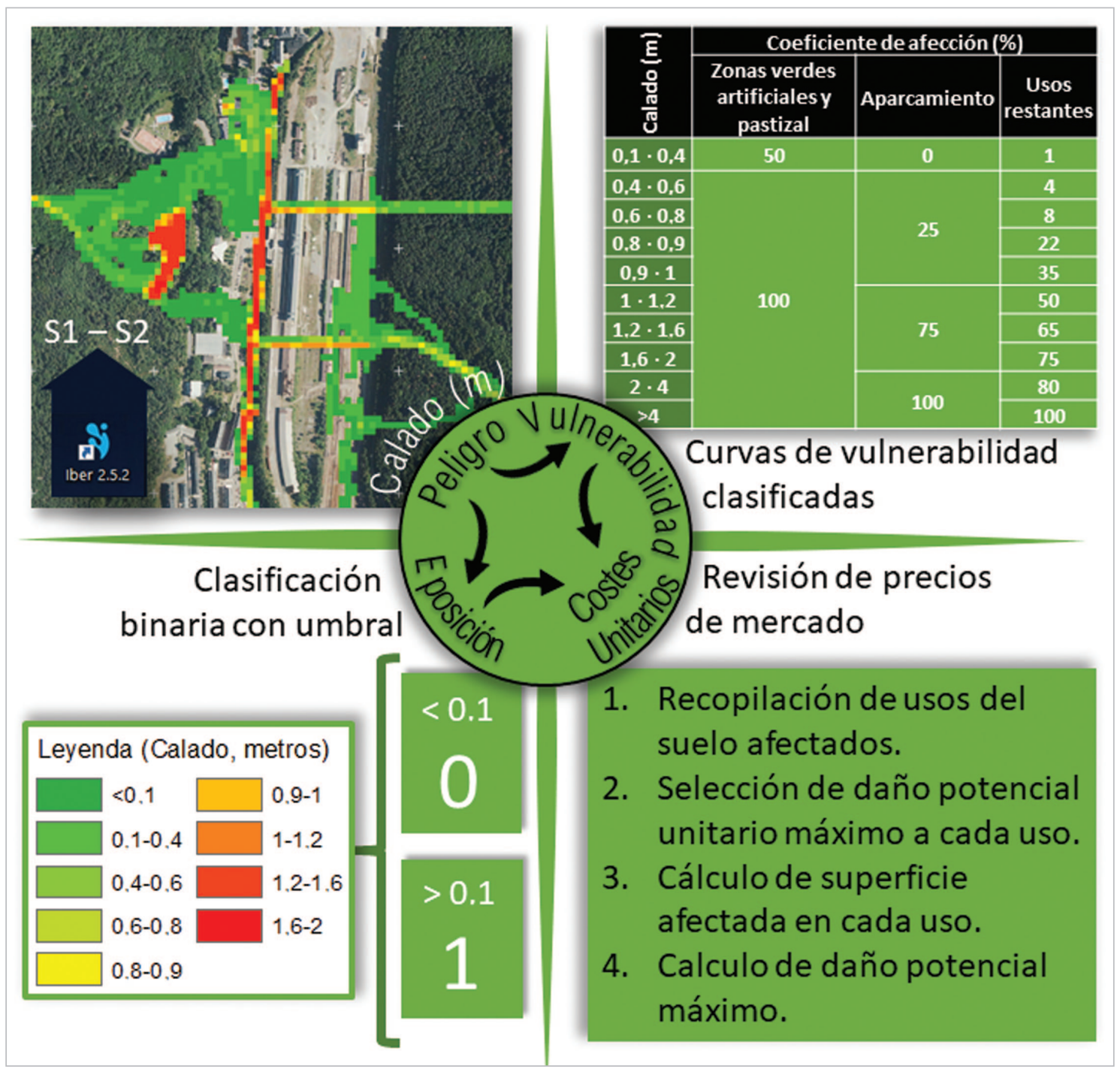

Figura 5. Aplicación de las variables del RC para el peligro de avenidas.

Fuente: elaboración propia.

de la Propuesta de Mínimos para la Metodología de Realización de los Mapas se Riesgo de Inundación (MAGRAMA, 2013). Este método, aquí empleado, requiere del calado y pocos datos adicionales para condensar las variables del RC en el uso de curvas de daños y daños potenciales en función de los usos del suelo (Fig. 5) (Gracia et al., 2014; Tachini et al., 2015; MAGRAMA, 2013; Fernández-Aracil, 2020). El calado ha sido obtenido con IBER (CEDEX et al., 2010; CEDEX et al., 2014), modificando en cada escenario: el MDT con las obras hidráulicas principales (Ayerbe, 1912b; Azpeitia, 1919); el cambio en el uso del suelo (Azpeitia, 1922b; Madoz, 1846-1850); y el caudal, variado en función de la Fórmula Racional y del método del Número de Curva por Fábregas (2015). En ambos escenarios se ha establecido un tipo de régimen crítico y se ha considerado exposición constante binaria mediante umbral. Obtenidos todos los datos se han aplicado las fórmulas 4-8. 


\subsubsection{Estimación de costes desconocidos}

Se han estimado los CM de ambos proyectos (formula 11) y los CE del proyecto hipotético. Los $\mathrm{CE}$ del proyecto hipotético se han obtenido con un proceso en dos etapas:

1. Recopilar la cantidad y características técnicas de los tipos de obras (Ayerbe, 1912b; Azpeitia, 1919; Pemán y Pérez-Soba, 2013; Fábregas Reigosa, 2015).

2. Estimar sus costes unitarios de construcción mediante revisión de precios de mercado.

Para estimar los CM se han empleado dos métodos:

1. El método del ratio, empleando ratios típicos entre el $2 \%$ y el $5 \%$ (Cappabianca et al., 2008; Paratscha et al., 2019).

2. La estimación de costes de mantenimiento anuales mediante planificación de actividades a posteriori. Para emplear el último método se requiere tomar datos en campo, imposible debido a las restricciones de movilidad (COVID19) . Para solventarlo se emplearon datos bibliográficos (Fábregas Reigosa, 2015) y datos facilitados por el gestor del MUP Nº406.

Con estos datos se ha seguido un proceso de tres etapas:

1. Seleccionar las obras donde se debe realizar mantenimiento (Cappabianca et al., 2008; Fábregas Reigosa, 2015).

2. Estimar los costes unitarios de mantenimiento mediante las características técnicas y la revisión de precios de mercado (Pemán y Pérez-Soba, 2013; Ballesteros et al., 2016).

3. Aplicar la fórmula 14.

\section{Resultados y discusión}

Los resultados son derivados de la defensa del MUP N ${ }^{\circ}$ 406. Se han obtenido indicadores comunes y resultados estandarizados por ha de MUP, que, debido a la incertidumbre del resultado, deben tomarse con cautela. Los cálculos fueron realizados para el escenario más favorable, menos favorable e intermedio respecto a los costes, contemplando todas las probabilidades de ocurrencia respecto a los PR y para intereses del $1 \%$ al $4 \%$. Todas las combinaciones obtuvieron resultados positivos, pero se ha analizado únicamente el escenario intermedio con probabilidades de ocurrencia coincidentes con probabilidades de excedencia (fórmula 6) y con un interés del $2 \%$, tratando al resultado de manera determinística. 


\subsection{Resultados económicos.}

Los resultados muestran un VAC asumible para el proyecto original, destacando el escaso porcentaje destinado a la repoblación forestal. Contrariamente, el VAC del proyecto hipotético refleja un proyecto $37 \%$ más costoso. Dentro de los costes destacan los costes medios por unidad de los diques, con rangos similares a los citados por Notaro y Paletto (2004) y Ballesteros et al. (2016). Respecto al VAB, los resultados muestran amplios márgenes de beneficio tanto para aludes como para avenidas, destacando las diferencias entre escenarios en los PR más bajos.

El valor protector obtenido es alto. Empleando un i del 1\% y estandarizando con los años de vida de la obra se puede comparar el resultado con otros casos (Notaro y Paletto, 2004). Paralelamente, los indicadores económicos restantes muestran resultados positivos. Todos los resultados se encuentran resumidos en la Tab. 4 y Fig. 6.

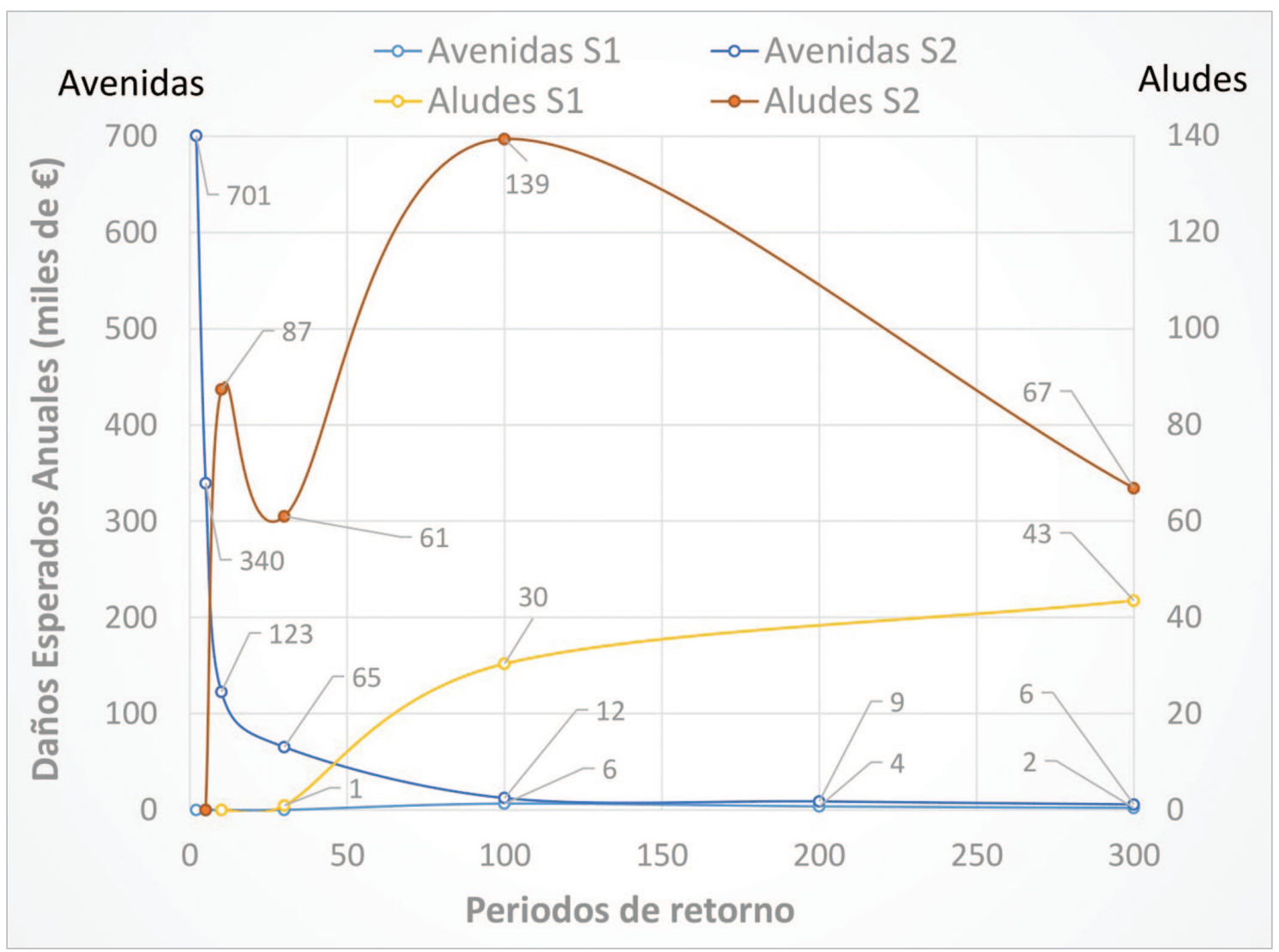

Figura 6. Daño Esperado para los peligros de aludes y avenidas en función de los PR.

Fuente: elaboración propia.

Para mejorar el carácter orientativo de los resultados, las sobreestimaciones y subestimaciones sobre el VAN final para el proyecto original se han categorizado asignándoles magnitud. Esto verifica que el VAN está ligeramente subestimado, pero ajustado a la realidad (Fig. 7). 
Tabla 4. Cuadro resumen resultados económicos.

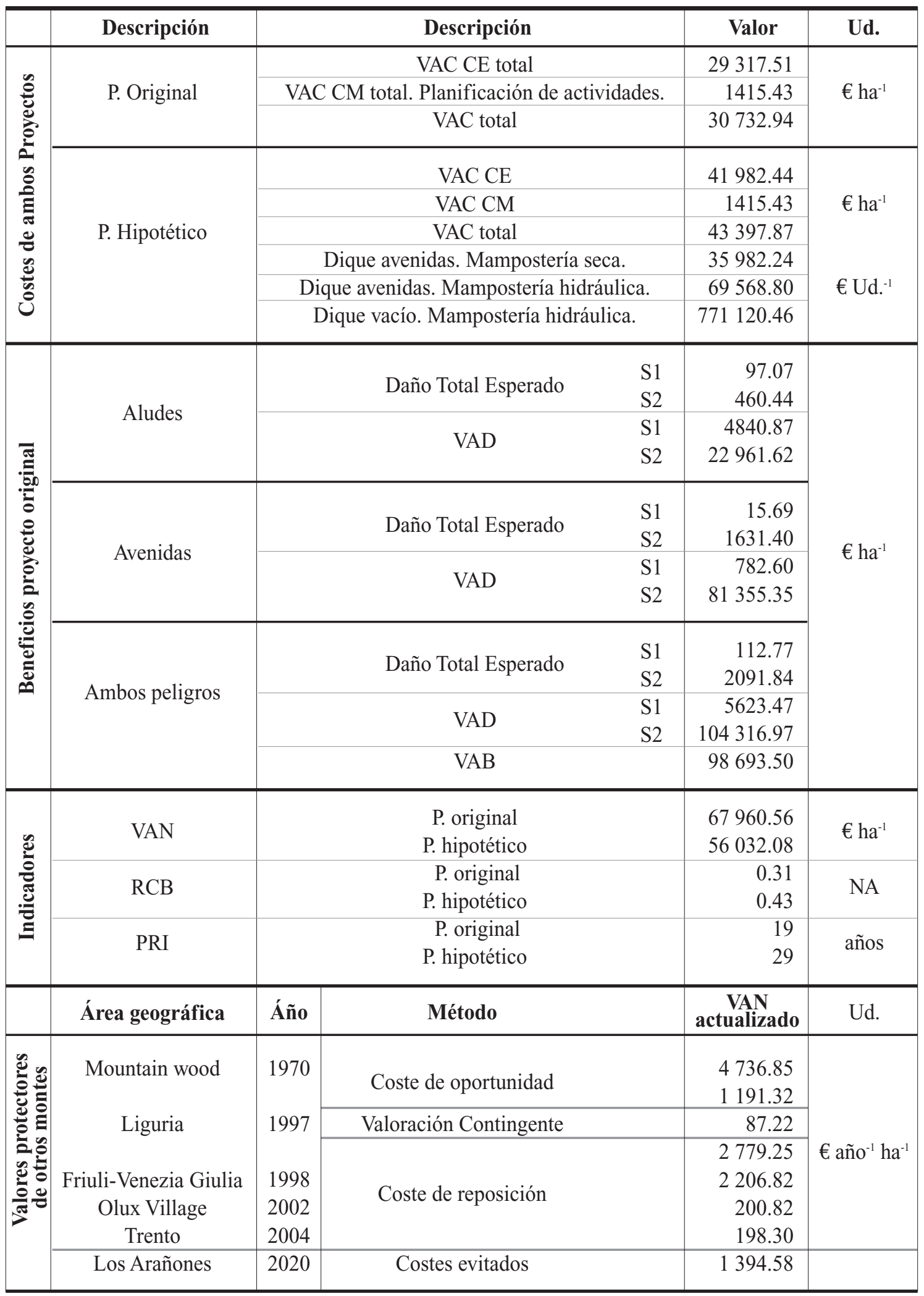




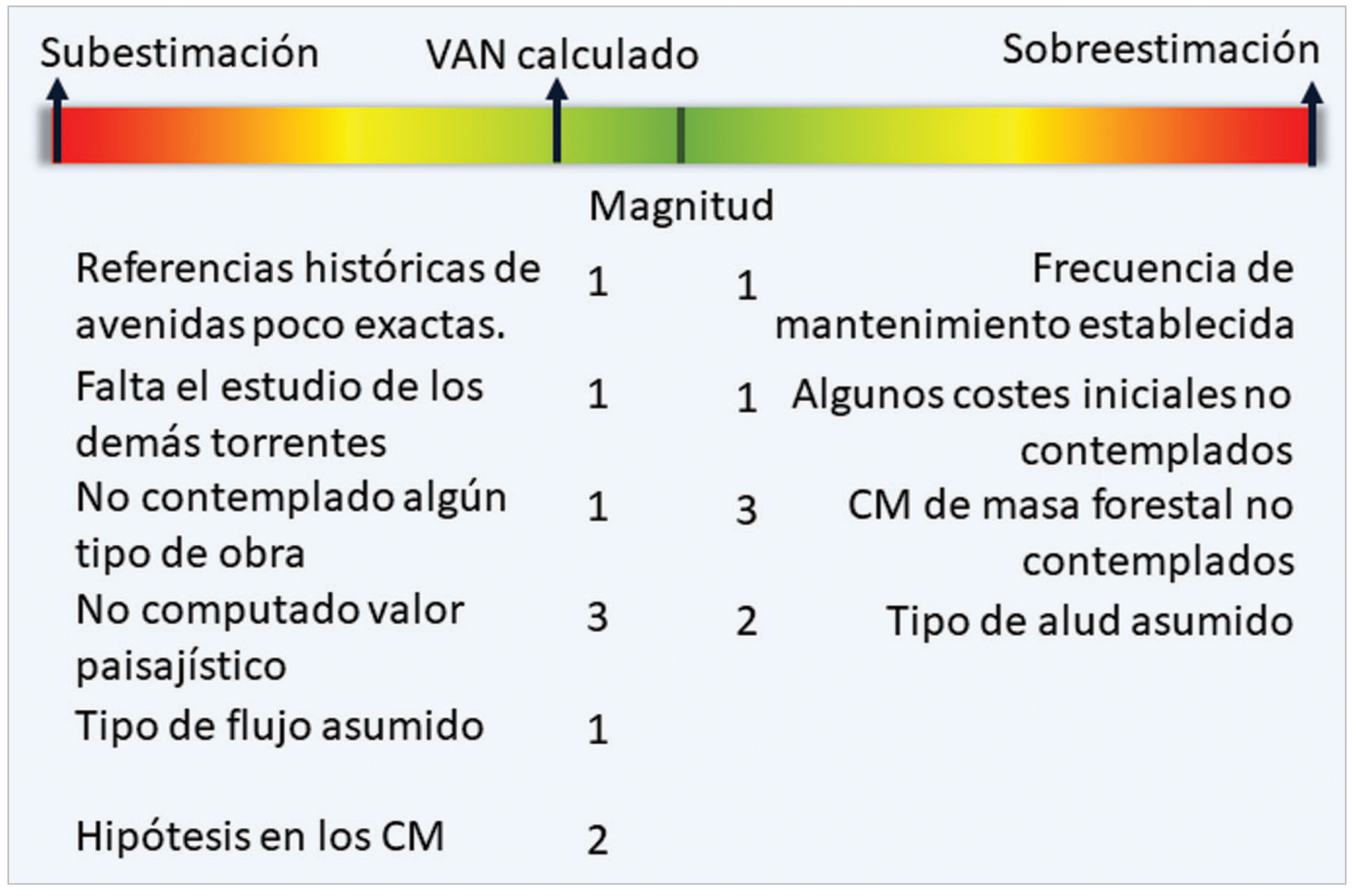

Figura 7. Subestimaciones y sobreestimaciones sobre el VAN calculado.

Fuente: elaboración propia.

\subsection{Resultados relativos al riesgo.}

Se han analizado los mapas de riesgo para aludes y avenidas y se ha obtenido la reducción del riesgo gráficamente (Wilhelm, 1998). En ambos escenarios destacan tres puntos:

(A) la iglesia de Canfranc.

(B) el edificio de viviendas situado en los número 1-4 de la Calle Valle del Aspe.

(C) la estación internacional. El punto A, más afectado históricamente, y sus protecciones actuales recientes generan afección indirecta en el punto $\mathrm{B}$, habitable y que presenta el mayor riesgo por aludes. El punto $\mathrm{C}$ se encuentra bien planificado y seguro, gracias a su localización y a las obras de defensa. No presenta riesgo, en contraposición al último estudio de riesgos (Geobrugg y Raymar, 2017). Los resultados muestran una clara reducción del riesgo (Fig. $8)$. Definir si este riesgo es aceptable depende de los actores implicados en Canfranc-Estación.

\subsection{Propuestas futuras}




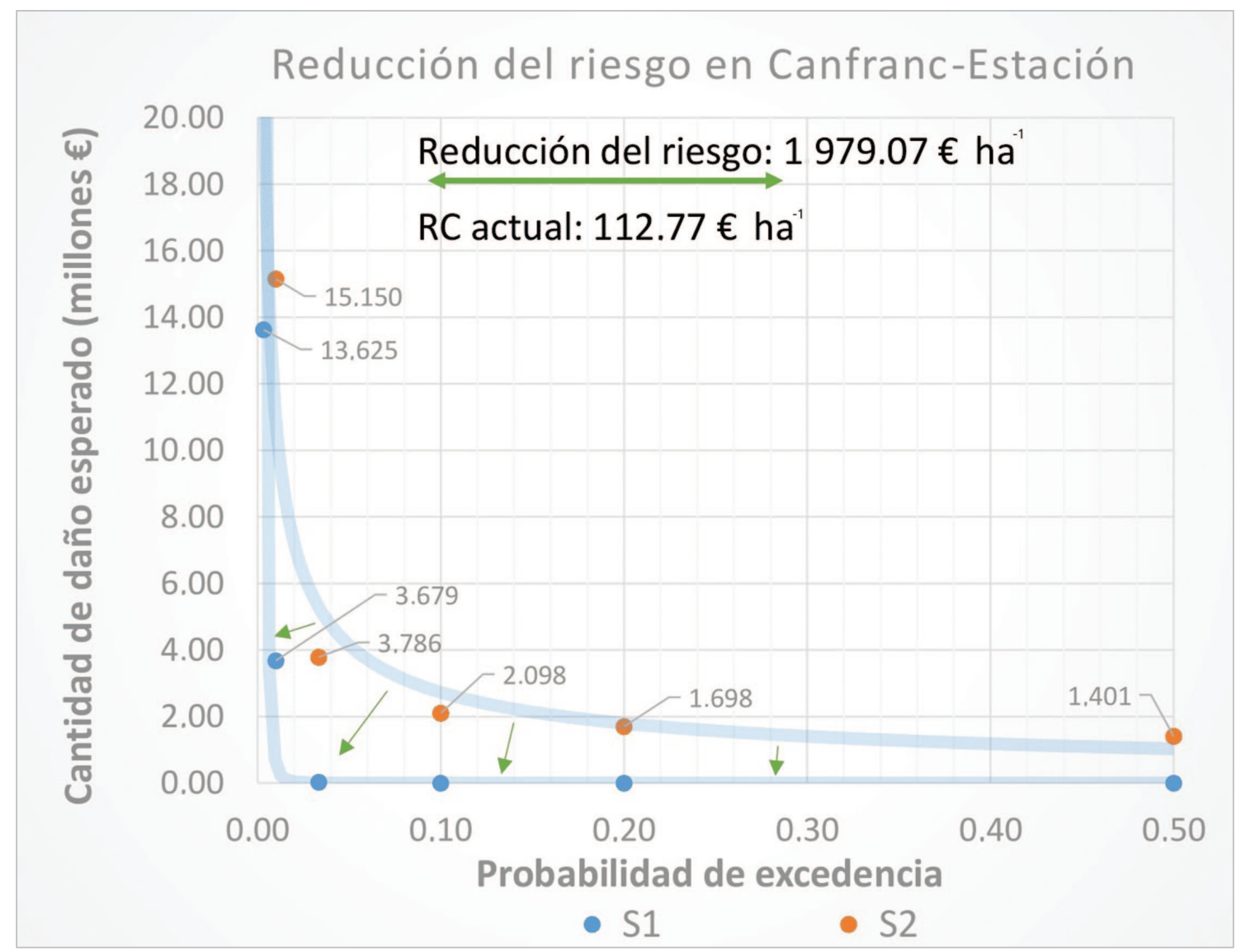

Figura 8. Reducción del RC y RC actual generado por las obras de defensa.

Fuente: elaboración propia.

Propuestas principales:

1. Planificación adaptada de actividades de mantenimiento que evite parches reactivos ya conocidos en la zona (Azpeitia, 1922a).

2. Completar datos de campo para mejorar los cálculos.

Propuestas adicionales:

1. Mantener el registro histórico de aludes y avenidas elaborado.

2. Aplicar la metodología a los torrentes menos relevantes incluyendo el valor paisajístico.

3. Completar el análisis estocástico de los resultados y aplicarlo en la gestión del riesgo. 


\section{Conclusiones}

Los indicadores económicos y el análisis del riesgo ratifican el gran valor protector de las obras — $67960.56 €$ ha $^{-1}$ — para Canfranc-Estación, capaces de reducir el riesgo en $1979.07 €$ ha $^{-1}$. Estas obras han reducido notablemente el riesgo de aludes y prácticamente en su totalidad el riesgo de avenidas. No obstante, pese a su gran eficacia, con una rentabilidad superior al $4 \%$ ejecutándose con un crédito exiguo, presentan algún detalle a corregir: las obras de defensa de la iglesia y la inversión en mantenimiento. Es importante además analizar la posible ocurrencia de eventos extremos ligados al cambio climático y su posible afección a las zonas urbanas, actuales y futuras, así como otras infraestructuras. Consecuentemente, los resultados abogan por elaborar una gestión adecuada del riesgo y del activo económico de las obras externalizado en este estudio.

\section{Agradecimientos y financiación}

A Fernando y José, por responder tan rápido en este contrarreloj. A Santiago Fábregas y Juan Ballesteros, cuyas charlas y datos aportaron calidad. A la Diputación General de Aragón, por su ayuda. A Inés González, por su ejemplo académico. A Inés Serrano, el mérito de los detalles bonitos es suyo.

\section{Bibliografía}

Andrade Fuentes, C.A.; 2019. Reconstrucción del alud de febrero de 2018 en la carretera BV4024, Coll de Pal, y definición y caracterización del correspondiente escenario de referencia de T100. Trabajo Fin de máster. Universidad Autónoma de Barcelona.

Ayerbe, B.; 1912b. El problema del Cuerpo de Ingenieros de Montes en la defensa de la estación y vía internacional del ferrocarril a Francia por Canfranc. Revista de Montes. 37, 305430.

Ayerbe, J.M.; 1952. El invierno de 1950-1951 en Los Arañones. Revista de Montes. 43, 23-25.

Ayerbe, P.; 1912a. Importancia y necesidad del Servicio Hidrológico-Forestal. Revista de Montes. 36 (839) 9-14.

Azpeitia, F.; 1919. Los trabajos hidrológico-forestales y la Estación Internacional de Los Arañones (Canfranc). Revista de Montes. 43, 773-847.

Azpeitia, F.; 1922a. Trabajos hidrológico-forestales para la defensa de Canfranc contra las avenidas del torrente. Los Meses. Revista de Montes. 46 (1073), 285-289.

Azpeitia, F.; 1922b. La Estación Internacional de los Arañones (Canfranc) y su defensa hidrológico-forestal. Revista de Montes. 46 (1074), 321-382.

Ballesteros Cánovas, J.A.; Stoffel, M.; Corona, C.; Schraml, K.; Gobiet, A.; Tani, S.; Sinabell, S.; Fuchs, S.; Kaitna, R.; 2016. Debris-flow risk analysis in a managed torrent based on a stochastic life-cycle performance. Science of The Total Environment, 557-558, 142-153. https://doi.org/10.1016/j.scitotenv.2016.03.036

Bartelt, P.; Bühler, Y.; Christen, M.; Deubelbeiss, Y.; Salz, M.; Schneider, M.; Schumacher, L.; 
2017. RAMMS: AVALANCHE User Manual. http://163.171.135.86/8107439.s21d8.faiusrd.com/61/ABUIABA9GAAg1MLk9AUo9ozYoQM.pdf?wsiphost=local\&wsrid_ta g=60edaebb_PSxbymdlMAD1sn6_24081-26105. Accessed. 14 June 2021.

Benito, M.T.; 1998. 1969-1999: Canfranc 30 años después. Espacio, Tiempo y Forma, Serie VI, Geografía. 11, 186-226.

Bernad, F.; 1913. Los torrentes de Canfranc. Revista de Montes. 37 (880), 629-637.

Cappabianca, F.; Barbolini, M.; Natale, L.; 2008. Snow avalanche risk assessment and mapping: A new method based on a combination of statistical analysis, avalanche dynamics simulation and empirically-based vulnerability relations integrated in a GIS platform. Cold Regions Science and Technology. 54 (3), 193-205. https://doi.org/10.1016/j.coldregions.2008.06.005

CEDEX, GEAMA.; FLUMEN, CIMNE.; 2010. IBER. Modelización bidimensional del flujo en lámina libre en aguas poco profundas. Manual básico de usuario. https://iberaula.es/54/ibermodel/downloads. Accessed. 14 June 2021.

CEDEX, GEAMA.; FLUMEN, CIMNE.; 2014. IBER. Modelización bidimensional del flujo en lámina libre en aguas poco profundas. Manual de referencia hidráulico. https://iberaula.es/ 54/iber-model/downloads. Accessed. 14 June 2021.

De Motes, J.M.; 2013. La inflación en España: un índice de precios de consumo, 1830-2012. Banco de España, Eurosistema, España.

Fábregas Reigosa, S.M.; 2015. Criterios de aproximación para medir la efectividad de las actuaciones hidrológico-forestales, en el control del fenómeno nivo-torrencial en las cuencas de montaña. Tesis Doctoral. Universidad Politécnica de Madrid.

Fábregas, F.; Fábregas, S.; 2001. Los Ingenieros de Montes y los trabajos de defensa frente a los aludes en la provincia de Huesca. Montes. 65, 74-79.

Fernández-Aracil, P;. 2020. El análisis coste-beneficio y la evaluación de los riesgos de inundación. Riesgo de inundación en España: análisis y soluciones para la generación de territorios resilientes. López Ortiz, M.I.; Melgarejo Moreno, J.; (eds.), Alicante, España, pp. 11531162.

Fernández-Santos, P.; Songini, G.; Hurtado, R.; Mintegui, J.A.; 2015. Delimitación de la zona amenazada por aludes, en el área dominada del torrente de Estiviellas (Canfranc-Estación, Huesca). Cuadernos de la Sociedad Española de Ciencias Forestales. 41, 115-130. https:// doi.org/10.31167/csef.v0i41.17382

Ferrando, E.L.; Cuchí Oterino, J.A.; 2004. Los aludes en el Alto Aragón. Lucas Mallada: revista de ciencias. 11,135-161.

Fuchs, S.; Thoeni, M.; Mcalpin, M.C.; Gruber, U.; Bründl, M.; 2007. Avalanche Hazard Mitigation Strategies Assessed by Cost Effectiveness Analyses and Cost Benefit Analyses- evidence from Davos, Switzerland. Natural Hazards, 41(1), 113-129. https://doi.org/10.1007/ s11069-006-9031-z

González, E.; 1928. Las obras de defensa de la Estación Internacional de Los Arañones. La Voz. 2356, 18.

Gracia, A.; Godé, L.; Crego, E.; Arrabal, M.A.; Guirado, V.; García, G.; Lobera, C.; González, S.; Martínez, E.; 2014. Riesgos y cuantificación de daños por inundación. 15.

Jonkman, S.N.; Van Gelder, P.; Vrijling, J.K.; 2003. An overview of quantitative risk measures for loss of life and economic damage. Journal of hazardous materials. 99(1), 1-30. https:// doi.org/10.1016/S0304-3894(02)00283-2

Leo, E.; Cuchí, J.A.; 2017. Los aludes en el Alto Aragón: tipología, zonas de peligro, daños y víctimas. Lucas Mallada: revista de ciencias. 19, 233-278. 
Madoz, P.; 1846-1850. Diccionario geográfico-estadístico-histórico de España y sus posesiones de ultramar. Establecimiento tipográfico de Madoz, P. y Sagasti, L.; Madrid, España, pp. 451-452.

MAGRAMA. 2013. Propuesta de mínimos para la metodología de realización de los mapas de riesgos de inundación. Ministerio de Agricultura, Alimentación y Medio Ambiente. https:// www.miteco.gob.es/es/agua/temas/gestion-de-los-riesgos-de-inundacion/propuesta-de-minimos-realizacion-mapas-deriesgo-2ciclo_tcm30-511333.pdf. Accessed. 14 June 2021.

Mintegui Aguirre, J.A.; Lenzi, M.A.; Robredo, J.C.; 2006. Movilización versus estabilización de los sedimentos en los cursos sometidos a la dinámica torrencial. Organismo Autónomo Parques Nacionales, Ministerio de Medio Ambiente, Madrid, España, pp. 1-143.

Mintegui Aguirre, J.A.; Robredo, J.C.; Huelin, P;. 2016. Estudio del manto de nieve y defensa en la montaña ante el peligro de aludes. Fundación Conde del Valle de Salazar, Madrid, España, pp. 1-276.

Notaro, S.; Paletto, A.; 2004. Economic evaluation of the protective function of mountain forests: a case study from the Italian Alps. Journal of Forests Economics. 11.

Notaro, S.; Paletto, A.; 2008. Natural disturbances and natural hazards in mountain forests: a framework for the economic valuation. Artículo de debate n. ${ }^{\circ}$ 8. Dipartimento di Economia, Università degli studi di Trento.

Oller, P.; Janeras, M.; De Buén, H.; Arnó, G.; García, C.; Christen, M.; Martínez, M.; 2009. Validación del programa de modelización numérica Aval-1D para el cálculo de aludes en los Pirineos Orientales. VII Simposio Nacional sobre Taludes y Laderas Inestables.

Paratscha, R.; Von Der Thannen, M.; Smutny, R.; Lampalzer, T.; Strauss, A.; Rauch, H.P.; 2019. Development of LCA benchmarks for Austrian torrent control structures. The International Journal of Life Cycle Assessment. 24 (11), 2035-2053. https://doi.org/10.1007/s11367019-01618-6

Pemán, J.; Pérez-Soba, I.; 2013. Los Ayerbe: tres ingenieros de Montes altoaragoneses y su aportación a la hidrología forestal española. Lucas Mallada: revista de ciencias. 15, 99-160.

Pierson, T.; Costa, J.Y.; Vancouver, W.; 1987. Debris Flows/avalanches: Process, Recognition, and Mitigation. Geological Society of America. Reviews in Engineering Geology, America, pp. 1-12. https://doi.org/10.1130/REG7-p1

Plan Bleu., 2016. Sustainable Forest Management. Socio-economic assessment of goods and services provided by Mediterranean forest ecosystems. https://planbleu.org/. Accessed. $14 \mathrm{Ju}-$ ne 2021.

Smith, G.A.; 1986. Coarse-grained nonmarine volcaniclastic sediment: Terminology and depositional process. Geological Society of America Bulletin. 97(1), 1-10. https://doi.org/10. 1130/0016-7606(1986)97\%3C1:CNVSTA\%3E2.0.CO;2

Statham, G.; Haegeli, P.; Greene, E.; Birkeland, K.; Israelson, C.; Tremper, B.; Stethem, C.; Mcmahon, B.; White, B.; Kelly, J.; 2018. A conceptual model of avalanche hazard. Natural Hazards, 90(2), 663-691. https://doi.org/10.1007/s11069-017-3070-5

Tachini, M.; Severo, D.L.; Cordero, A.; Momo, M.R.; Silva, H.S.; 2015. Associated Damage Assessment to Flooding in City Blumenau. Ninth International Conference on Complex, Intelligent, and Software Intensive Systems, IEEE Explore, 402-407. https://doi.org/10.1109/ CISIS.2015.77

Wilhelm, C.; 1998. Quantitative risk analysis for evaluation of avalanche protection projects. Proceedings of the Anniversary Conference, 288-293. 